\title{
Amorphous Hafnium-Indium-Zinc Oxide Semiconductor Thin Film Transistors
}

\author{
Sheng-Po Chang and San-Syong Shih \\ Institute of Microelectronics and Department of Electrical Engineering, Center for Micro/Nano Science and Technology, \\ Advanced Optoelectronic Technology Center, National Cheng Kung University, Tainan 70101, Taiwan \\ Correspondence should be addressed to Sheng-Po Chang, changsp@mail.ncku.edu.tw
}

Received 14 March 2012; Revised 17 June 2012; Accepted 18 June 2012

Academic Editor: Gong Ru Lin

Copyright ( $\odot 2012$ S.-P. Chang and S.-S. Shih. This is an open access article distributed under the Creative Commons Attribution License, which permits unrestricted use, distribution, and reproduction in any medium, provided the original work is properly cited.

\begin{abstract}
We reported on the performance and electrical properties of co-sputtering-processed amorphous hafnium-indium-zinc oxide $(\alpha$ HfIZO) thin film transistors (TFTs). Co-sputtering-processed $\alpha$-HfIZO thin films have shown an amorphous phase in nature. We could modulate the In, Hf, and Zn components by changing the co-sputtering power. Additionally, the chemical composition of $\alpha$-HfIZO had a significant effect on reliability, hysteresis, field-effect mobility $(\mu \mathrm{FE})$, carrier concentration, and subthreshold swing (S) of the device. Our results indicated that we could successfully and easily fabricate $\alpha$-HfIZO TFTs with excellent performance by the co-sputtering process. Co-sputtering-processed $\alpha$-HfIZO TFTs were fabricated with an on/off current ratio of $\sim 10^{6}$, higher mobility, and a subthreshold slope as steep as $0.55 \mathrm{~V} / \mathrm{dec}$.
\end{abstract}

\section{Introduction}

Thin-film transistors (TFTs) serve as circuit switches between a backlight unit and a flat panel display. Therefore, the desired properties of TFTs for flat panel display application include high-field-effect mobility, excellent subthreshold swing, excellent stability, high optical transparency, and a high on/off current ratio. In addition, TFTs manufactured by the cosputtering process can meet these requirements with an easier and lower-temperature manufacturing process. As such, cosputtering-processed amorphous oxide semiconductor thin films have been attracting extreme attention as active layers in TFTs that exhibit remarkable device performance [1-9]. Previously reported thin films with $\mathrm{ZnO}$ as the active layer have a polycrystalline structure with columnar grains $[10,11]$, even those deposited at room temperature. These grain boundaries result in several problems such as significant leakage current, poor electrical performance over large areas, and instability. Therefore, recently developed TFTs with amorphous zinc-indium-tin oxide (ZITO) or amorphous indium-gallium-zinc oxide (IGZO) thin films as the active layer have been reported. Amorphous IGZO was developed as a channel layer, and the results showed that the film had a field-effect-mobility of $23 \mathrm{~cm}^{2} / \mathrm{Vs}$, a threshold voltage of $-3 \mathrm{~V}$, and a subthreshold gate voltage swing (SS) of $0.75 \mathrm{~V} /$ decade [12]. Despite the fact that these oxide semiconductors exhibit remarkable electrical properties such as high-field-effect mobility, low threshold voltage, and excellent subthreshold swing, their working instability under negative bias illumination is the main reason for the degradation in device performance and for the decrease in device lifetime. In this paper, we report the performance and electrical properties of cosputtering-processed amorphous hafnium-indium-zinc oxide $(\alpha$-HfIZO) TFTs. We desire solving the problem of instability under negative bias illumination by using hafnium (Hf) to replace gallium $(\mathrm{Ga})$ in IGZO as the active layer because $\mathrm{Hf}$ has a higher affinity for oxygen than gallium. In the future, we want to perform reliability tests. Furthermore, our greatest advantage is the ability to modulate the In, Hf, and $\mathrm{Zn}$ components by changing the cosputtering power. The modulation of the Hf content in the HfIZO devices could control the crystalline structure of the thin film and increase device reliability. Thus, the physical and electrical properties and the reliability of 


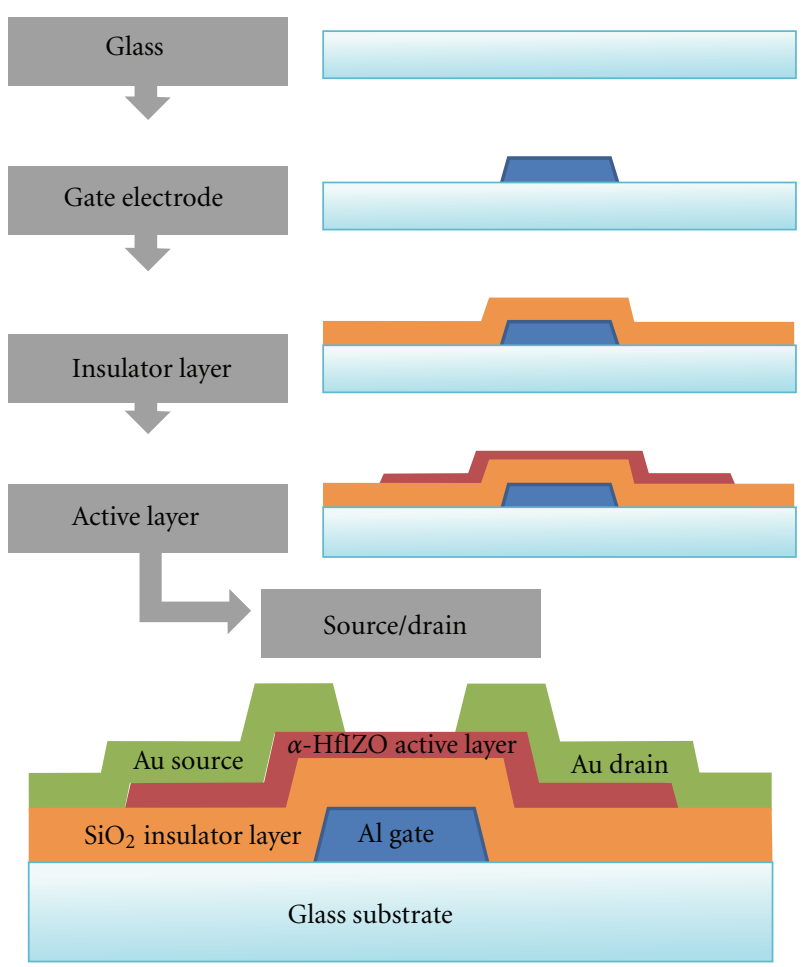

FIGURE 1: Device processing steps used in this study.

HfIZO TFTs, which show outstanding electrical properties and stability under bias stresses, will also be discussed.

\section{Experiment}

The schematic diagram in Figure 1 shows the processing steps and the cross-section of the bottom-gate structure of the $\alpha$-HfIZO TFTs on a glass substrate. The entire device underwent a third shadow mask process. First, the aluminum gate electrode was deposited onto the glass substrate through a shadow mask using thermal evaporation at room temperature. The $200 \mathrm{~nm}$ thick $\mathrm{SiO}_{2}$ insulator layer was deposited over the entire glass substrate area by plasma-enhanced chemical vapor deposition (PECVD). Then, $\mathrm{In}_{2} \mathrm{O}_{3}, \mathrm{ZnO}$, and $\mathrm{HfO}_{2}$ were co-sputtered through the second shadow mask without heating the substrate to produce a $50 \mathrm{~nm}$ thick active layer. Before deposition of the active layer, we evacuated the sputtering chamber to a base pressure of $3 \times 10^{-6}$ Torr. During the active layer sputtering, the chamber pressure was kept at $10 \mathrm{mTorr}$. The flow rates of the $\mathrm{O}_{2} / \mathrm{Ar}$ gas mixture was $45 / 55 \mathrm{sccm}$ using two RF guns and one DC gun. $\mathrm{In}_{2} \mathrm{O}_{3}, \mathrm{HfO}_{2}$, and $\mathrm{ZnO}$ targets were installed on the RF magnetron sputtering and DC magnetron sputtering system. The DC gun was maintained at $20 \mathrm{~V}$, and the RF gun was maintained at $100 \mathrm{~V}$. The full targets were $99.99 \mathrm{wt} \%$ and 3 in size. Finally, a $100 \mathrm{~nm}$ thick Au source and drain electrodes were deposited by thermal evaporation on the active layer through the final shadow mask. The channel length $L$ and the channel width $W$ of the completed $\alpha$-HfIZO TFTs were 200 and $200 \mu \mathrm{m}$, respectively.
The current-voltage $(I-V)$ characteristics of the fabricated $\alpha$ HfIZO TFTs were measured using an HP4156 semiconductor parameter analyzer. The high-performance $\alpha$-HfIZO thinfilm transistors were fabricated without postannealing.

\section{Results and Discussion}

An energy-dispersive spectrometer (EDS) was used to confirm the elemental composition of the $\alpha$-HfIZO active layer. Figure 2 and Table 1 show the EDS spectrum of the composited elements and the proportion of elements in the $\alpha$-HffZO active layer. It was found that Hf, In, Zn, and oxide were present in the active layer region [13]. The analysis of the electrical properties of the drain current to source-drain voltage $\left(I_{D}-V_{D S}\right)$ characteristic curves is shown in Figure 3. The $I_{\mathrm{D}}-V_{\mathrm{D}}$ characteristics of the $\alpha$-IGZO TFT were measured in the dark. As the drain-source voltage $\left(V_{\mathrm{DS}}\right)$ swept from 0 to $2 \mathrm{~V}$, the gate-source voltage $\left(V_{\mathrm{GS}}\right)$ swept from 0 to $2 \mathrm{~V}$ in steps of $0.4 \mathrm{~V}$. The drain current to source-gate voltage ( $I_{\mathrm{D}^{-}}$ $V_{\mathrm{GS}}$ ) characteristics curves of the $\alpha$-HfIZO TFTs are shown in Figure 4 . The source-gate voltage was increased significantly to record the transfer characteristics of the TFTs at a drainsource voltage $\left(V_{\mathrm{DS}}\right)$ of $2 \mathrm{~V}$ by increasing $V_{\mathrm{GS}}$ from -0.5 to $2 \mathrm{~V}$, as shown in Figure 4. It was known that $\alpha$-HfIZO is an $n$ type material due to the positive gate voltages. The threshold voltage $\left(V_{\text {th }}\right)$ values were calculated by extrapolation from the plot of the square root of $I_{\mathrm{DS}}$ versus $V_{\mathrm{GS}}$ curve in the saturation region. From the graph, we can obtain the $V_{\text {th }}$ value of $0.95 \mathrm{~V}$. This was extracted using the following equation for a field-effect transistor:

$$
I_{\mathrm{D}}=\frac{\mu_{\mathrm{FE}} C_{i}}{2} \frac{W}{L}\left(V_{\mathrm{GS}}-V_{\mathrm{th}}\right)^{2}, \quad \text { for } V_{\mathrm{DS}}>V_{\mathrm{GS}}-V_{\mathrm{th}},
$$

where $C_{i}$ is the gate capacitance per unit area, $W$ is the active layer width $(1000 \mu \mathrm{m}), L$ is the active layer length $(100 \mu \mathrm{m})$, and $\mu_{\mathrm{FE}}$ is the field-effect mobility of TFTs that was calculated using the following equation:

$$
\mu_{\mathrm{FE}}=\frac{L \mathrm{gm}}{W C_{\mathrm{SiO} 2} V_{\mathrm{DS}}},
$$

where $C_{\mathrm{SiO} 2}$ is the specific capacitance per unit area of the silicon dioxide $\left(C_{\mathrm{SiO} 2}: 1.72 \times 10^{8} \mathrm{~F} / \mathrm{cm}^{2}\right)$, gm is the transconductance, and $W / L$ is the aspect ratio of the active layer. In this graph, we obtained a current on/off $\left(I_{\text {on }} / I_{\text {off }}\right)$ ratio of $\sim 10^{6}$; the device had a high $\mu_{\mathrm{FE}}$ of $32.6 \mathrm{~cm}^{2} /$ Vs. Therefore, it is particularly promising for low-power-consumption active-matrix liquid crystal display (AMLCD) or for activematrix organic light emitting diode (AMOLED) products. In addition, the interface defect densities $\left(N_{t}\right)$ of the TFTs were calculated from the subthreshold swing values using the following equation:

$$
N_{t}=\left[\frac{\operatorname{SS} \log (e)}{(k T / q)}-1\right] \frac{C_{i}}{q}
$$

where $q$ is the charge of the electron, $k$ is the Boltzmann's constant, and $C_{i}$ is the gate capacitance per unit area. The $S$ value depends on the number of defects in the interface 


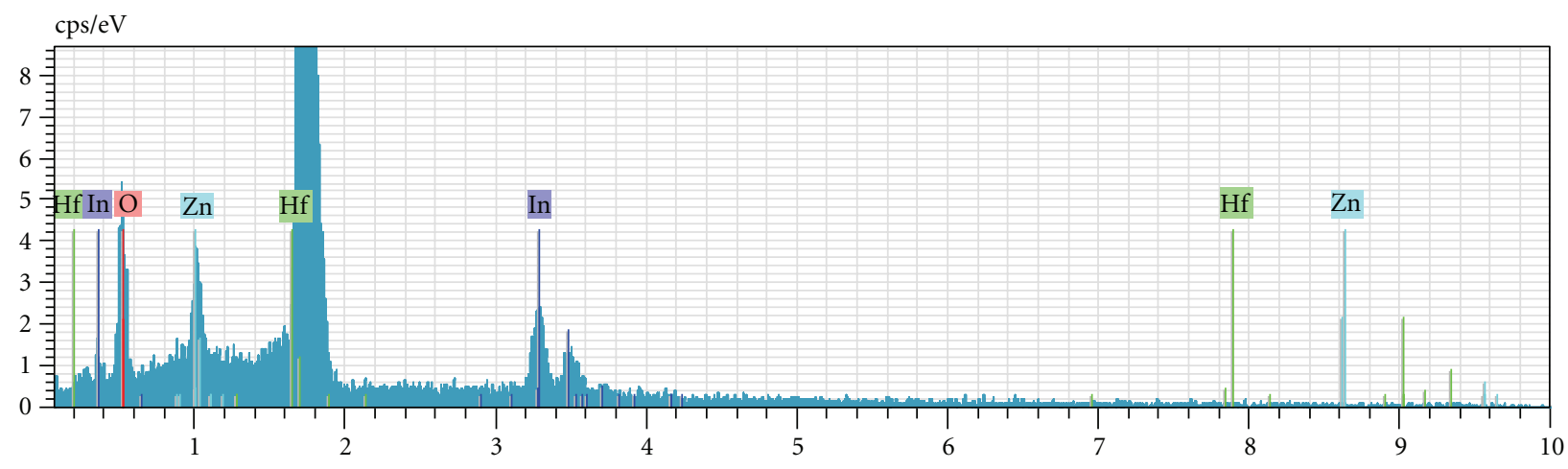

$(\mathrm{KeV})$

FIGURE 2: Energy-dispersive spectrometer (EDS) spectrum of the $\alpha$-HfIZO active layer.

TABLE 1: Proportion of elements in the $\alpha$-HfIZO active layer.

\begin{tabular}{|c|c|c|c|c|c|c|}
\hline El & AN & Series & unn. $C$ [wt.\%] & norm. $C$ [wt.\%] & Atom. $C$ [at.\%] & Error [wt.\%] \\
\hline $\mathrm{O}$ & 8 & K-series & 13.35 & 13.01 & 57.13 & 2.9 \\
\hline Hf & 72 & M-series & 59.17 & 57.65 & 22.69 & 2.7 \\
\hline In & 49 & L-series & 25.13 & 24.48 & 14.98 & 1.1 \\
\hline \multirow[t]{2}{*}{$\mathrm{Zn}$} & 30 & L-series & 4.98 & 4.85 & 5.21 & 0.4 \\
\hline & & Total: & 102.62 & 100.00 & 100.00 & \\
\hline
\end{tabular}

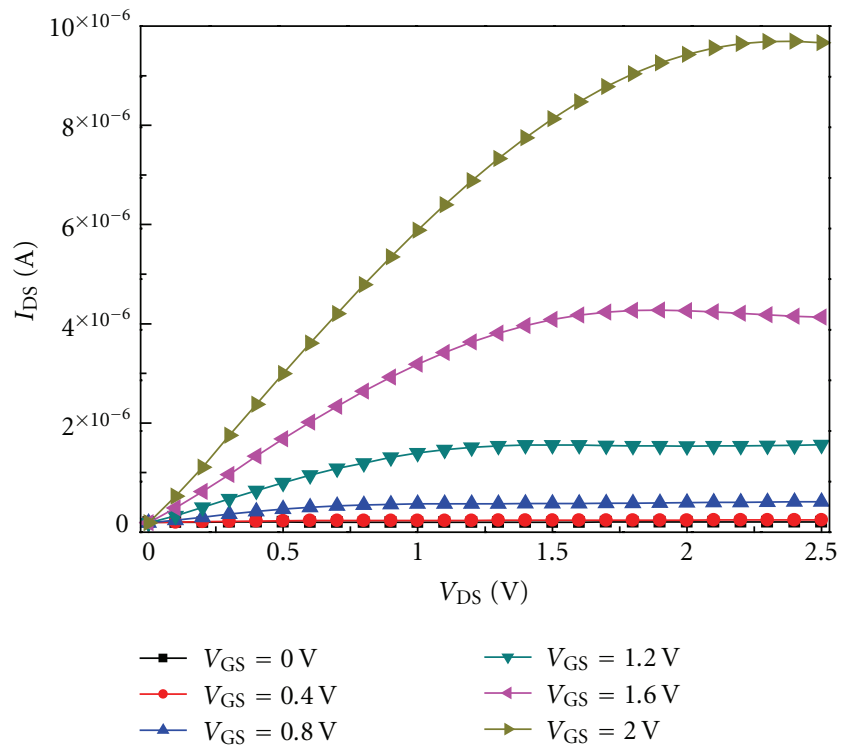

FIGURE 3: Drain current to source-drain voltage $\left(I_{\mathrm{D}}-V_{\mathrm{DS}}\right)$ characteristics curves.

between the active layer and $\mathrm{SiO}_{2}$ insulator layer from the aforementioned equation. Therefore, we obtained the $S$ value of 0.55 , and $N_{t}$ was $8.8 \times 10^{11} \mathrm{~cm}^{-2}$, which indicate promising electrical properties for TFT devices. The parameters calculated from the equation of transfer characteristics are listed in Table 2. We compare the ZITO, IGZO, GZO, and HfIZO TFTs electrical properties. The HfIZO TFTs have a sufficient on-to-off ratio of $\sim 10^{6}$ compared with IGZO TFTs $\left(\sim 10^{5}\right)$

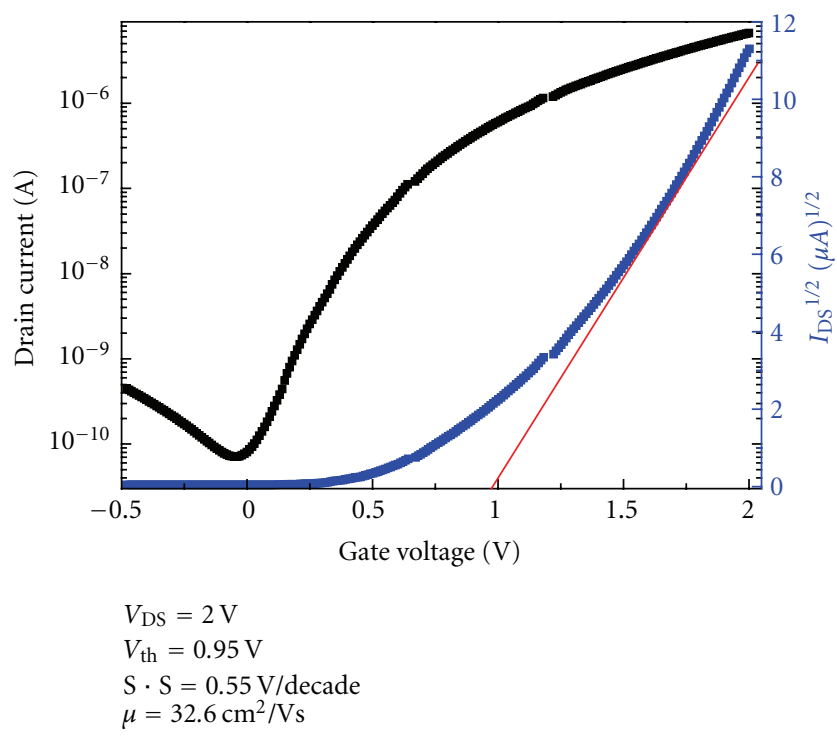

FIgure 4: The drain current to source-gate voltage $\left(I_{\mathrm{D}}-V_{\mathrm{GS}}\right)$ characteristics curves.

and ZITO TFTs $\left(\sim 10^{6}\right)$. The HfIZO TFTs have a mobility of $32.6 \mathrm{~cm}^{2} / \mathrm{Vs}$ compared with IGZO TFTs of $23 \mathrm{~cm}^{2} / \mathrm{Vs}$. Therefore, HfIZO is a more appropriate material for TFT switching.

\section{Conclusion}

We proposed an easier, lower-temperature cosputtering process. We successfully fabricated TFTs with amorphous HfIZO 
TABLE 2: Parameters calculated from the equation of transfer characteristics.

\begin{tabular}{lccccc}
\hline Composition & $\mu_{\mathrm{FE}}\left(\mathrm{cm}^{2} / \mathrm{Vs}\right)$ & $V_{\mathrm{th}}(\mathrm{V})$ & $I_{\mathrm{ON}} / I_{\mathrm{OFF}}$ & $\mathrm{SS}(\mathrm{V} / \mathrm{decade})$ & 0.55 \\
HfIZO & 32.6 & 0.95 & $\sim 10^{6}$ & 0.75 & $8.8 \times 10^{11}$ \\
IGZO & 23 & -3 & $\sim 10^{5}$ & 2.25 & $1.2 \times 10^{12}$ \\
ZITO & 70 & 4.5 & $\sim 10^{6}$ & 1.25 & $3.9 \times 10^{12}$ \\
GZO & 1.63 & -0.6 & $\sim 10^{6}$ & $2.1 \times 10^{12}$ \\
\hline
\end{tabular}

thin film as the active layer by the cosputtering process on a glass substrate; this exhibited remarkable device performance such as a higher field-effect mobility, lower $V_{\text {th }}$, higher subthreshold swing, and enhanced mode operation. Compared with IGZO and ZITO TFTs, HfIZO TFTs have outstanding performance. Therefore, $\alpha$-HfIZO thin films can be used as the active layer for TFTs. We believe $\alpha$-HIZO TFT is a good candidate for low-power-consumption products.

\section{Acknowledgments}

This work was supported by the National Science Council under Contract NSC 100-2221-E-006-168. This work was also supported in part by the Center for Frontier Materials and Micro/Nano Science and Technology, National Cheng Kung University, Taiwan. This work was also supported in part by the Advanced Optoelectronic Technology Center, National Cheng Kung University, under projects from the Ministry of Education.

\section{References}

[1] B. Du Ahn, H. S. Shin, G. H. Kim, J. S. Park, and H. J. Kim, "A novel amorphous InGaZnO thin film transistor structure without source/drain layer deposition," Japanese Journal of Applied Physics, vol. 48, no. 3, Article ID 03B019, 4 pages, 2009.

[2] Y. K. Moon, S. Lee, W. S. Kim et al., "Improvement in the bias stability of amorphous indium gallium zinc oxide thinfilm transistors using an $\mathrm{O}_{2}$ plasma-treated insulator," Applied Physics Letters, vol. 95, no. 1, Article ID 013507, 3 pages, 2009.

[3] N. C. Su, S. J. Wang, and A. Chin, "High-performance InGaZnO thin-film transistors using HfLaO gate dielectric," IEEE Electron Device Letters, vol. 30, no. 12, pp. 1317-1319, 2009.

[4] K. Nomura, H. Ohta, A. Takagi, T. Kamiya, M. Hirano, and H. Hosono, "Room-temperature fabrication of transparent flexible thin-film transistors using amorphous oxide semiconductors," Nature, vol. 432, no. 7016, pp. 488-492, 2004.

[5] E. M. C. Fortunato, P. M. C. Barquinha, A. C. M. B. G. Pimen tel et al., "Fully transparent $\mathrm{ZnO}$ thin-film transistor produced at room temperature," Advanced Materials, vol. 17, no. 5, pp. 590-594, 2005.

[6] J. S. Park, K. Kim, Y. G. Park, Y. G. Mo, H. D. Kim, and J. K. Jeong, "Novel ZrInZnO thin-film transistor with excellent stability," Advanced Materials, vol. 21, no. 3, pp. 329-333, 2009.

[7] J. M. Lee, I. T. Cho, J. H. Lee, W. S. Cheong, C. S. Hwang, and H. I. Kwon, "Comparative study of electrical instabilities in top-gate InGaZnO thin film transistors with $\mathrm{Al}_{2} \mathrm{O}_{3}$ and $\mathrm{Al}_{2} \mathrm{O}_{3} / \mathrm{SiN}_{x}$ gate dielectrics," Applied Physics Letters, vol. 94, no. 22, Article ID 222112, 3 pages, 2009.
[8] W. Lim, E. A. Douglas, S. H. Kim et al., "High mobility InGaZnO ${ }_{4}$ thin-film transistors on paper," Applied Physics Letters, vol. 94, no. 7, Article ID 072103, 3 pages, 2009.

[9] J. S. Park, T. W. Kim, D. Stryakhilev et al., "Flexible full color organic light-emitting diode display on polyimide plastic substrate driven by amorphous indium gallium zinc oxide thinfilm transistors," Applied Physics Letters, vol. 95, no. 1, Article ID 013503, 3 pages, 2009.

[10] R. L. Huffman, "Zno-channel thin-film transistors: channel mobility," Journal of Applied Physics, vol. 95, no. 10, pp. 58135819, 2004.

[11] F. M. Hossain, J. Nishii, S. Takagi et al., "Modeling and simulation of polycrystalline $\mathrm{ZnO}$ thin-film transistors," Journal of Applied Physics, vol. 94, no. 12, pp. 7768-7777, 2003.

[12] C. J. Chiu, S. P. Chang, and S. J. Chang, "High-performance a-igzo thin-film transistor using $\mathrm{Ta}_{2} \mathrm{O}_{5}$ gate dielectric," IEEE Electron Device Letters, vol. 31, no. 11, pp. 1245-1247, 2010.

[13] C. J. Kim, S. Kim, J. H. Lee et al., "Amorphous hafniumindium-zinc oxide semiconductor thin film transistors," Applied Physics Letters, vol. 95, no. 25, Article ID 252103, 3 pages, 2009. 

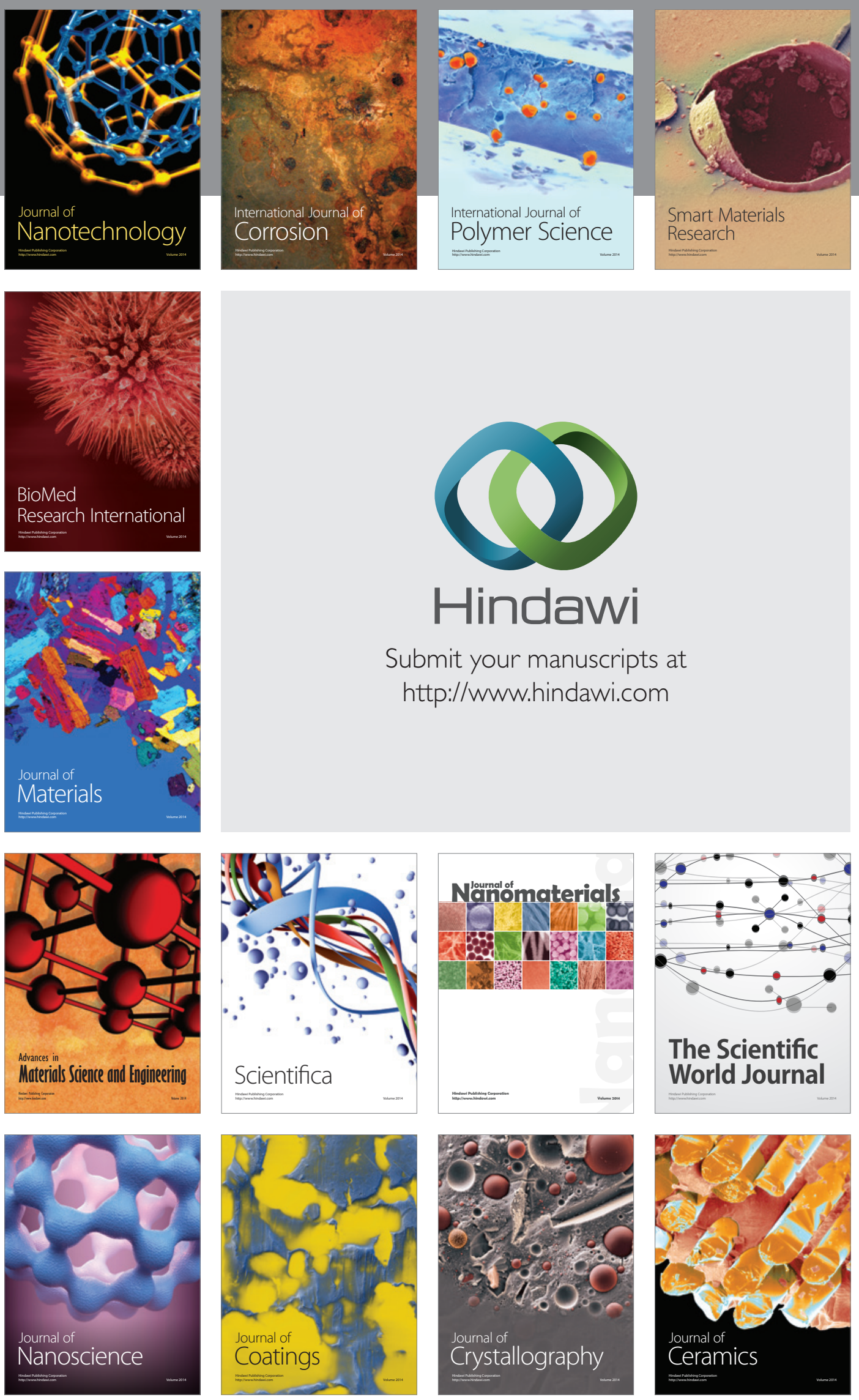

The Scientific World Journal

Submit your manuscripts at

http://www.hindawi.com

\section{World Journal}

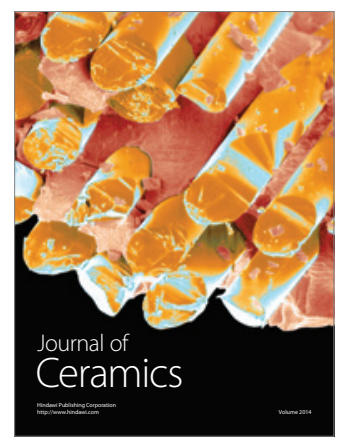

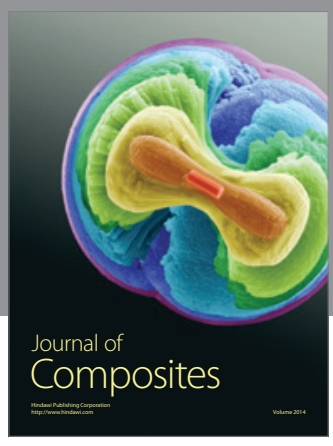
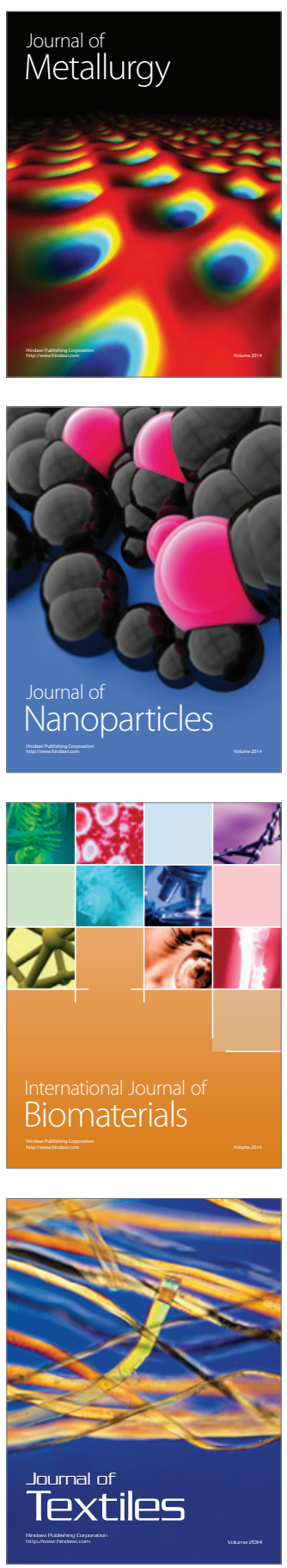